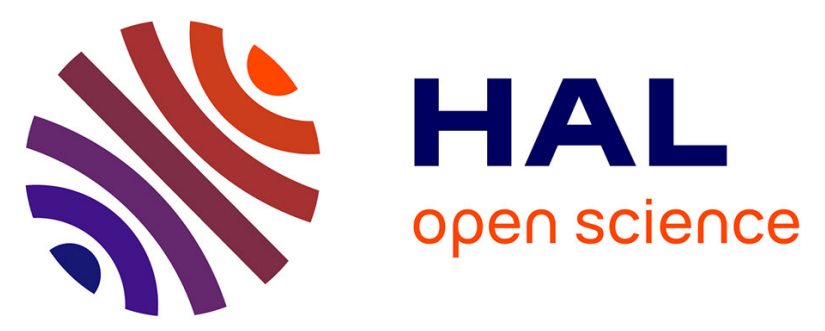

\title{
The substrate of Greatwall kinase, Arpp19, controls mitosis by inhibiting protein phosphatase $2 \mathrm{~A}$.
}

Aicha Gharbi-Ayachi, Jean-Claude Labbé, Andrew Burgess, Suzanne

Vigneron, Jean-Marc Strub, Estelle Brioudes, Alain Van-Dorsselaer, Anna

Castro, Thierry Lorca

\section{To cite this version:}

Aicha Gharbi-Ayachi, Jean-Claude Labbé, Andrew Burgess, Suzanne Vigneron, Jean-Marc Strub, et al.. The substrate of Greatwall kinase, Arpp19, controls mitosis by inhibiting protein phosphatase 2A.. Science, 2010, 330 (6011), pp.1673-7. 10.1126/science.1197048 . hal-00556511

\section{HAL Id: hal-00556511 https://hal.science/hal-00556511}

Submitted on 25 Jun 2021

HAL is a multi-disciplinary open access archive for the deposit and dissemination of scientific research documents, whether they are published or not. The documents may come from teaching and research institutions in France or abroad, or from public or private research centers.
L'archive ouverte pluridisciplinaire HAL, est destinée au dépôt et à la diffusion de documents scientifiques de niveau recherche, publiés ou non, émanant des établissements d'enseignement et de recherche français ou étrangers, des laboratoires publics ou privés. 
Aicha Gharbi-Ayachi, et al.

Science 330, 1673 (2010);

DOI: $10.1126 /$ science. 1197048

This copy is for your personal, non-commercial use only.

If you wish to distribute this article to others, you can order high-quality copies for your colleagues, clients, or customers by clicking here.

Permission to republish or repurpose articles or portions of articles can be obtained by following the guidelines here.

The following resources related to this article are available online at www.sciencemag.org (this infomation is current as of December 16, 2010 ):

Updated information and services, including high-resolution figures, can be found in the online version of this article at:

http://www.sciencemag.org/content/330/6011/1673.full.html

Supporting Online Material can be found at:

http://www.sciencemag.org/content/suppl/2010/12/14/330.6011.1673.DC1.html

This article cites 11 articles, 4 of which can be accessed free:

http://www.sciencemag.org/content/330/6011/1673.full.html\#ref-list-1

This article has been cited by 1 articles hosted by HighWire Press; see:

http://www.sciencemag.org/content/330/6011/1673.full.html\#related-urls

This article appears in the following subject collections:

Cell Biology

http://www.sciencemag.org/cgi/collection/cell_biol 
Ensa are dephosphorylated, presumably by protein phosphatase(s) other than PP2A-B558, such as PP1 $(29,30)$. The threshold for activation of Gwl and entry into mitosis depends on the balance of activities of these as-yet unidentified phosphatases and that of MPF.

\section{References and Notes}

1. D. O. Morgan, in The Cell Cycle: Principles of Control (Primers in Biology) (New Science Press, London, 2007), p. 297.

2. A. Lindqvist, V. Rodríguez-Bravo, R. H. Medema, J. Cell Biol. 185, 193 (2009).

3. A. Kumagai, W. G. Dunphy, Cell 70, 139 (1992).

4. P. R. Mueller, T. R. Coleman, W. G. Dunphy, Mol. Biol. Cell 6, 119 (1995).

5. S. Y. Kim, E. J. Song, K. J. Lee, ]. E. Ferrell Jr., Mol. Cell. Biol. 25, 10580 (2005)

6. S. L. Harvey, A. Charlet, W. Haas, S. P. Gygi, D. R. Kellogg, Cell 122, 407 (2005).

7. G. Pal, M. T. Paraz, D. R. Kellogg, J. Cell Biol. 180, 931 (2008).

8. S. Mochida, S. Ikeo, J. Gannon, T. Hunt, EMBO J. 28, 2777 (2009).
9. P. V. Castilho, B. C. Williams, S. Mochida, Y. Zhao, M. L. Goldberg, Mol. Biol. Cell 20, 4777 (2009).

10. S. Mochida, T. Hunt, Nature 449, 336 (2007).

11. J. Yu, Y. Zhao, Z. Li, S. Galas, M. L. Goldberg, Mol. Cell 22, 83 (2006).

12. S. Vigneron et al., EMBO J. 28, 2786 (2009)

13. M. Goldberg, personal communication.

14. P. Cohen, A. Knebel, Biochem. J. 393, 1 (2006)

15. Methods are available as supporting material on Science Online.

16. J. A. Girault, A. Horiuchi, E. L. Gustafson, N. L. Rosen, P. Greengard, J. Neurosci. 10, 1124 (1990).

17. A. Virsolvy-Vergine et al., Proc. Natl. Acad. Sci. U.S.A. 89, 6629 (1992).

18. L. Gros et al., Diabetologia 45, 703 (2002)

19. Single-letter abbreviations for the amino acid residues are as follows: A, Ala; C, Cys; D, Asp; E, Glu; F, Phe; G, Gly; H, His; I, Ile; K, Lys; L, Leu; M, Met; N, Asn; P, Pro; Q, Gln; R, Arg; S, Ser; T, Thr; V, Val; W, Trp; and Y, Tyr.

20. I. Dulubova et al., J. Neurochem. 77, 229 (2001).

21. E. Kinoshita, E. Kinoshita-Kikuta, K. Takiyama, T. Koike, Mol. Cell. Proteomics 5, 749 (2006).

22. A. W. Murray, Cell Biol. 36, 581 (1991).

23. K. Ohsumi, T. M. Yamamoto, M. Iwabuchi, Methods Mol. Biol. 322, 445 (2006)
24. G. Goshima et al., Science 316, 417 (2007); 10.1126/science.1141314.

25. J. R. Von Stetina et al., Development 135, 3697 (2008).

26. D. Drummond-Barbosa, A. C. Spradling, Dev. Biol. 266, 310 (2004).

27. A. Burgess et al., Proc. Natl. Acad. Sci. U.S.A. 107, 12564 (2010).

28. S. Mangan, A. Zaslaver, U. Alon, J. Mol. Biol. 334, 197 (2003).

29. K. Ishii, K. Kumada, T. Toda, M. Yanagida, EMBO J. 15, 6629 (1996).

30. ]. Q. Wu et al., Nat. Cell Biol. 11, 644 (2009).

31. We thank H. Mahbubani, ]. Kirk, and L. Egbuniwe for care of frogs and other members of the laboratory for advice and reagents, especially ]. Gannon for the antibody to doubly phosphorylated Thr $^{14}$-Tyr ${ }^{15}$ of Cdk1 (CP 3.2). S.M. was supported by a fellowship from the Japan Society for the Promotion of Science.

Supporting Online Material

www.sciencemag.org/cgi/content/full/330/6011/1670/DC1 Methods

Figs. S1 to S4

27 July 2010; accepted 13 October 2010

10.1126/science.1195689

\section{The Substrate of Greatwall Kinase, Arpp19, Controls Mitosis by Inhibiting Protein Phosphatase 2A}

\begin{abstract}
Aicha Gharbi-Ayachi, ${ }^{1}$ Jean-Claude Labbé, ${ }^{1}$ Andrew Burgess, ${ }^{1}$ Suzanne Vigneron, ${ }^{1}$ Jean-Marc Strub, ${ }^{2}$ Estelle Brioudes, ${ }^{1}$ Alain Van-Dorsselaer, ${ }^{2}$ Anna Castro, ${ }^{1_{*}} \dagger$ Thierry Lorca ${ }^{1 *} \dagger$

Initiation and maintenance of mitosis require the activation of protein kinase cyclin B-Cdc2 and the inhibition of protein phosphatase 2A (PP2A), which, respectively, phosphorylate and dephosphorylate mitotic substrates. The protein kinase Greatwall (Gwl) is required to maintain mitosis through PP2A inhibition. We describe how Gwl activation results in PP2A inhibition. We identified cyclic adenosine monophosphate-regulated phosphoprotein 19 (Arpp19) and $\alpha$-Endosulfine as two substrates of Gwl that, when phosphorylated by this kinase, associate with and inhibit PP2A, thus promoting mitotic entry. Conversely, in the absence of Gwl activity, Arpp19 and $\alpha$-Endosulfine are dephosphorylated and lose their capacity to bind and inhibit PP2A. Although both proteins can inhibit PP2A, endogenous Arpp19, but not $\alpha$-Endosulfine, is responsible for PP2A inhibition at mitotic entry in Xenopus egg extracts.
\end{abstract}

$\mathrm{M}$ itotic entry and exit are controlled by activation and inactivation of the cyclin B-Cdc2 protein kinase (1). However, maintained phosphorylation of mitotic proteins also requires inhibition of the protein phosphatase 2A (PP2A), the main phosphatase that dephosphorylates mitotic substrates (although a role of protein phosphatase 1 has also been reported in Xenopus egg extracts) (2-5).

The protein kinase Greatwall regulates PP2A activity during mitosis $(2,4,6)$. Depletion of Gwl

${ }^{1}$ Universités Montpellier 2 et 1 , Centre de Recherche de Biochimie Macromoléculaire, CNRS UMR 5237, IFR 122, 1919 Route de Mende, 34293 Montpellier cedex 5, France. ${ }^{2}$ Institut Pluridisciplinaire Hubert Curien, Strasbourg Cedex, France.

*To whom correspondence should be addressed. E-mail: anna.castro@crbm.cnrs.fr (A.C.); thierry.lorca@crbm.cnrs. fr (T.L.)

†These authors contributed equally to this work. from mitotic egg extracts induces mitotic exit, whereas depletion of Gwl from interphase egg extracts prevents mitotic entry (2-4). These phenotypes, as well as the phenotypes observed in Gwl knockdown human cells (O), are mediated by PP2A. Although Gwl and PP2A do interact, direct phosphorylation of $\mathrm{PP} 2 \mathrm{~A}$ by this kinase has not been observed $(2,4)$, suggesting that Gwl may inhibit PP2A through an intermediary protein. We used biochemical fractionation of cytostatic factor (CSF) meiotic arrested Xenopus egg extracts and in vitro phosphorylation by Gwl to identify substrates of this kinase.

We fractionated proteins from interphase extracts on a heparin column. Eluted proteins were precipitated with ammonium sulfate (50 to $70 \%$ ), resuspended, and fractionated by gel filtration. Samples from the fractions were phosphorylated in vitro with a hyperactive form of human Gwl (K72M mutant) (7), and the phosphoryl- ated bands were analyzed by mass spectrometry. Maximal phosphorylation was observed in the gel filtration fractions corresponding to molecular sizes of 30 to $70 \mathrm{kD}$ (Fig. 1A, fraction 3) and 20 to $50 \mathrm{kD}$ (Fig. 1A, fraction 4). These two fractions increased phosphorylation of a band of $20 \mathrm{kD}$. Forty different proteins were detected in this band by mass spectrometry, among them the cyclic adenosine monophosphate-regulated phosphoprotein 19 (Arpp19), a protein that strongly resembles the small protein $\alpha$-Endosulfine. The functions of Arpp19 and $\alpha$-Endosulfine are unclear $(8,9)$; however, oocytes from Drosophila with mutant $\alpha$-Endosulfine have a prolonged prophase and fail to progress to metaphase (10). Cdc2 isolated from these oocytes has normal kinase activity, but the oocytes show a reduced amount of in vivo phosphorylation of mitotic substrates, a phenotype reminiscent of the one observed in Gwl-depleted Xenopus egg extracts (4). Although by mass spectrometry we identified peptides specific to Arpp19 but not for $\alpha$-Endosulfine (fig. S1), their sequence similarity indicates that both may be Gwl substrates. We therefore tested glutathione $S$-transferase fusion proteins made from Arpp19 and $\alpha$-Endosulfine, as possible substrates of Gwl. Both proteins were phosphorylated in vitro by $\mathrm{Gwl}$ (Fig. 1B). The endogenous proteins were present in small amounts in Xenopus egg extracts, particularly Arpp19, which was very difficult to detect (Fig. 1C). The analysis of the protein sequences of Arpp19 and $\alpha$-Endosulfine revealed the presence of seven potential serine or threonine phosphorylation sites conserved in both proteins (Fig. 1D). We made individual mutants of Arpp19 in which serine (S) or threonine $(\mathrm{T})$ was mutated to alanine (A) and tested whether they were phosphorylated in vitro by Gwl. All mutants were phosphorylated by Gwl except mutant S62A. Similarly, mutation of this conserved serine of human $\alpha$-Endosulfine to alanine (S67A) also prevented the phosphorylation of this protein by Gwl (Fig. 
1D). These results indicate that this serine is the Gwl phosphorylation site in both Arpp19 and $\alpha$-Endosulfine.

We analyzed the effects of Arpp19 and $\alpha$ Endosulfine added to interphase Xenopus egg extracts. Either of these two proteins, if first phosphorylated in vitro by Gwl, promoted rapid mitotic entry as shown by the dephosphorylation of tyrosine 15 of $\mathrm{Cdc} 2$ and the phosphorylation of the mitotic substrates, followed by a subsequent exit from mitosis as indicated by the degradation of cyclin A and cyclin B2 (Fig. 1E). In a second experiment, to easily visualize mitotic entry, we prevented cyclin degradation by depleting the anaphase-promoting complex constituent Cdc27. In these extracts, the addition of phosphorylated Arpp19 or $\alpha$-Endosulfine pro- moted a rapid entry into mitosis and the maintenance of the mitotic state. Nonphosphorylated protein or the Arpp19 S62A or the $\alpha$-Endosulfine S67A phosphorylation mutants had no effect (Fig. 2A).

Gwl mediates entry into mitosis through the inhibition of PP2A, and so we suspected that Arpp19 and $\alpha$-Endosulfine could mediate mitotic entry by directly inhibiting PP2A. However, we could not exclude the possibility that these two proteins also regulate cyclin $\mathrm{B}-\mathrm{Cdc} 2$ activity to promote entry into mitosis. To determine whether Arpp19 or $\alpha$-Endosulfine could induce mitotic entry through the regulation of cyclin B$\mathrm{Cdc} 2$ activity, we depleted $\mathrm{Cdc} 25$ in interphase egg extracts and analyzed whether the two proteins still induced mitotic entry (11). We also de- pleted $\mathrm{Cdc} 27$ to prevent cyclin degradation and to easily visualize mitotic entry in these extracts. Either phospho-Arpp19 or phospho- $\alpha$-Endosulfine induced entry into mitosis in these extracts where only cyclin A-Cdc2 appeared to be active (Fig. 2B). Thus, Arpp19 and $\alpha$-Endosulfine are substrates of Gwl that promote mitotic entry, likely through the regulation of PP2A but not through the regulation of cyclin $\mathrm{B}-\mathrm{Cdc} 2$.

Accordingly, when Gwl was depleted from interphase egg extracts, the addition of $\alpha$-Endosulfine and Arpp19 still induced mitotic entry, further indicating that they act downstream of Gwl (Fig. 2C). These two proteins promoted entry into mitosis in Gwl-depleted interphase extracts only when they were thio-phosphorylated, indicating that when Gwl is present, dephosphoryl-
A

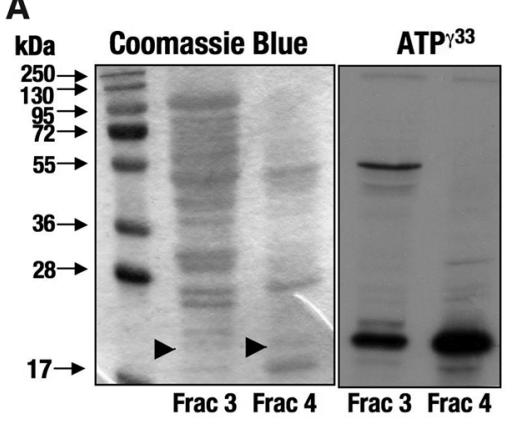

B

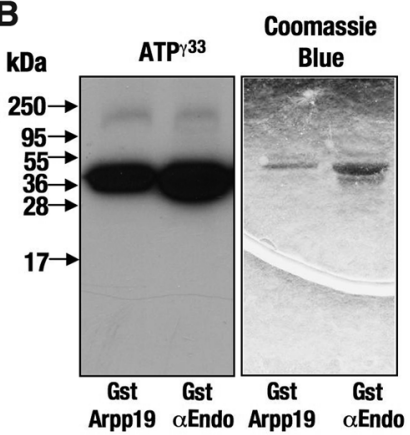

E
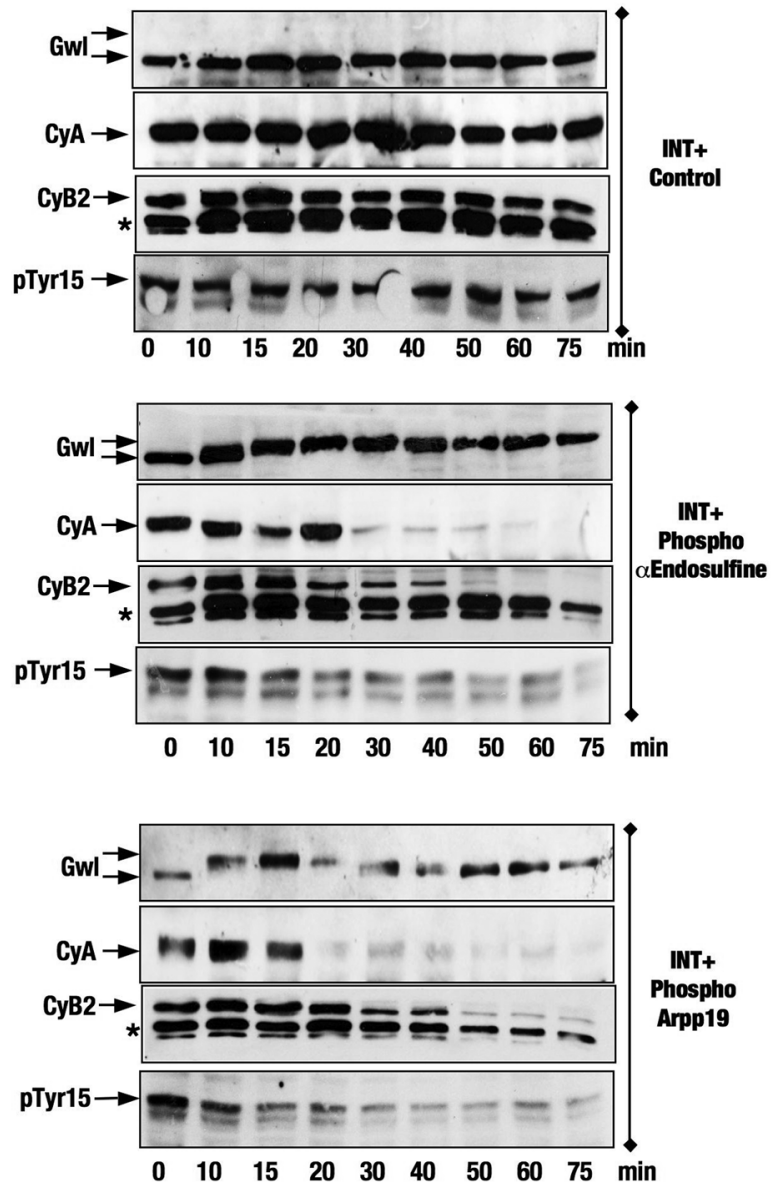

$\mathrm{C}_{\mathrm{kD}}$
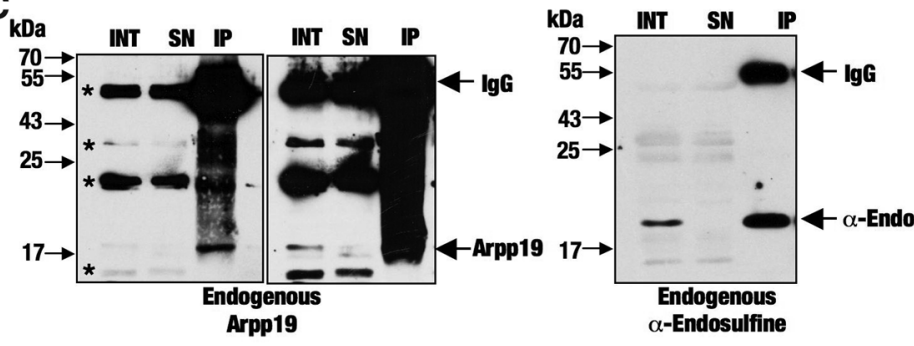

D

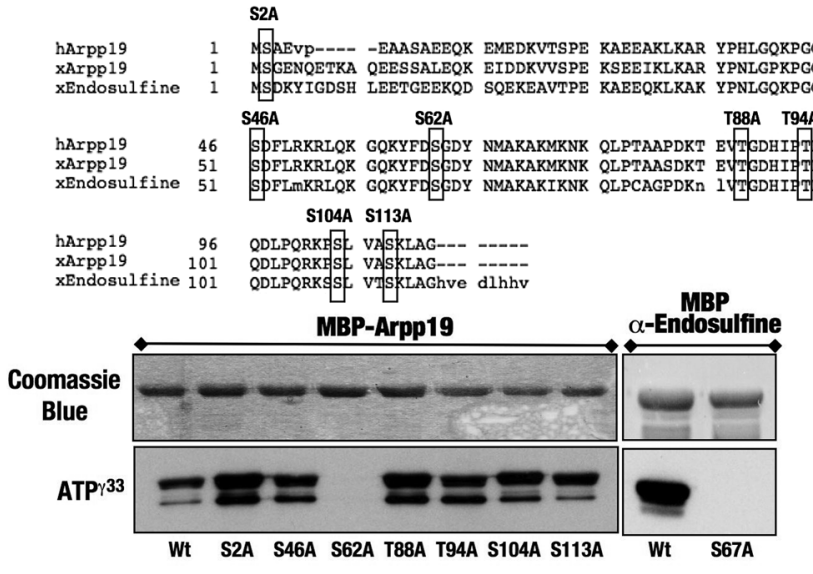

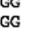


ation of these two substrates is counterbalanced by endogenous Gwl, whereas when $\mathrm{Gwl}$ is absent, these two proteins are rapidly dephosphorylated and do not promote or maintain the mitotic state.

Moreover, the addition of thio-phosphorylated Arpp19 or $\alpha$-Endosulfine to Gwl-depleted CSF extracts also prevented mitotic exit (Fig. 2D), demonstrating that Arpp19 and $\alpha$-Endosulfine are phosphorylated by Gwl and act downstream of this kinase to promote mitotic entry, possibly by directly inhibiting PP2A. Accordingly, phosphorylation of the Gwl-specific site on Arpp19 and $\alpha$-Endosulfine (S62/S67) decreased simultaneously with dephosphorylation of Gwl at mitotic exit (Fig. 2E), and it increased again concomitantly with the phosphorylation of this kinase at mitotic entry (Fig. 2F).

To determine whether Arpp19 or $\alpha$-Endosulfine could bind and inhibit PP2A, we incubated glutathione $S$-transferase (GST)-Arpp19 and GST$\alpha$-Endosulfine with interphase or with mitotic egg extracts and tested for association of PP2A with the fusion proteins. GST- $\alpha$-Endosulfine (moderately) and GST-Arpp19 (very weakly) bound PP2A in interphase egg extracts, in which Gwl is inactive. However, the binding increased (17 times for GST-Arpp19 and twice for GST- $\alpha$-Endosulfine) in mitotic egg extracts, in which Gwl is fully active, suggesting that the association of these two proteins with the A and $\mathrm{C}$ subunits of $\mathrm{PP} 2 \mathrm{~A}$ is increased by their Gwl-dependent phosphorylation. In support of this idea, the mutation to alanine of the Gwl phosphorylation site S62 in Arrp19 and S67 in $\alpha$-Endosulfine prevented the binding of either protein to PP2A. However, this association appears not to be exclusively regulated by Gwl-dependent phosphorylation because the $\alpha$-Endosulfine mutant D66A did not bind
A
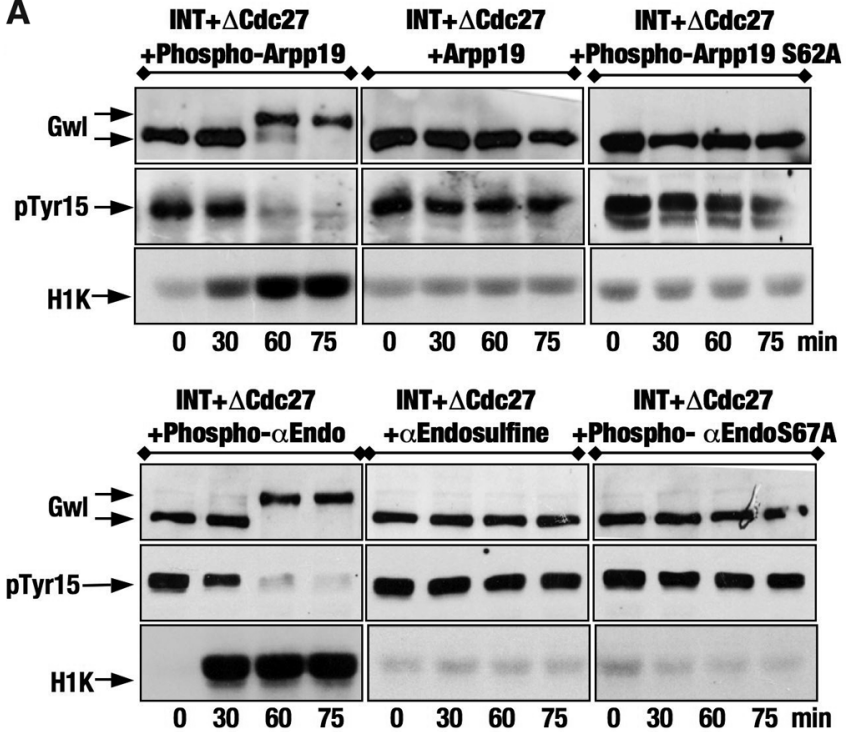

D

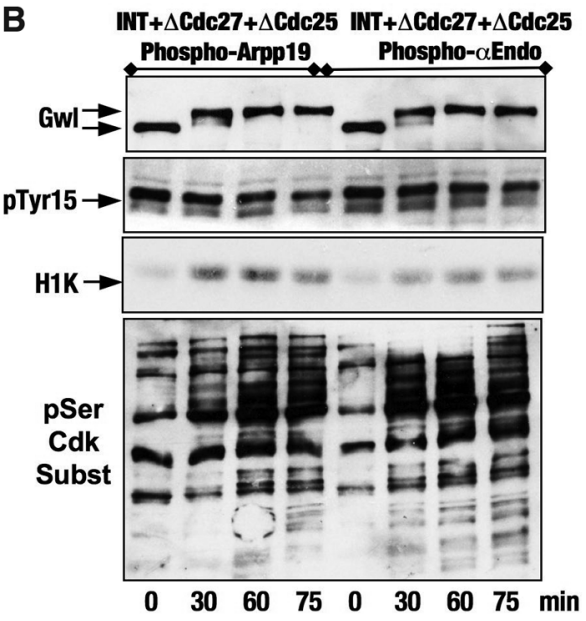

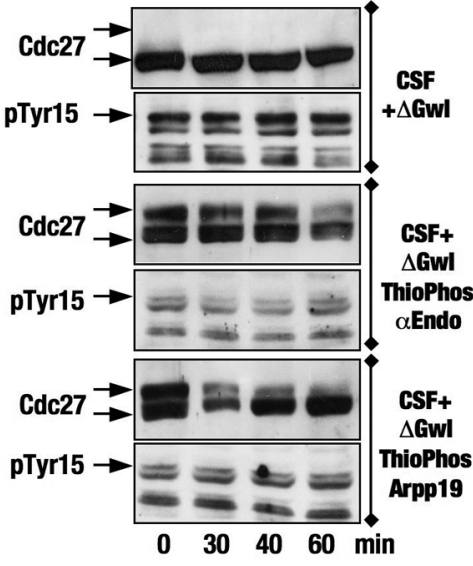
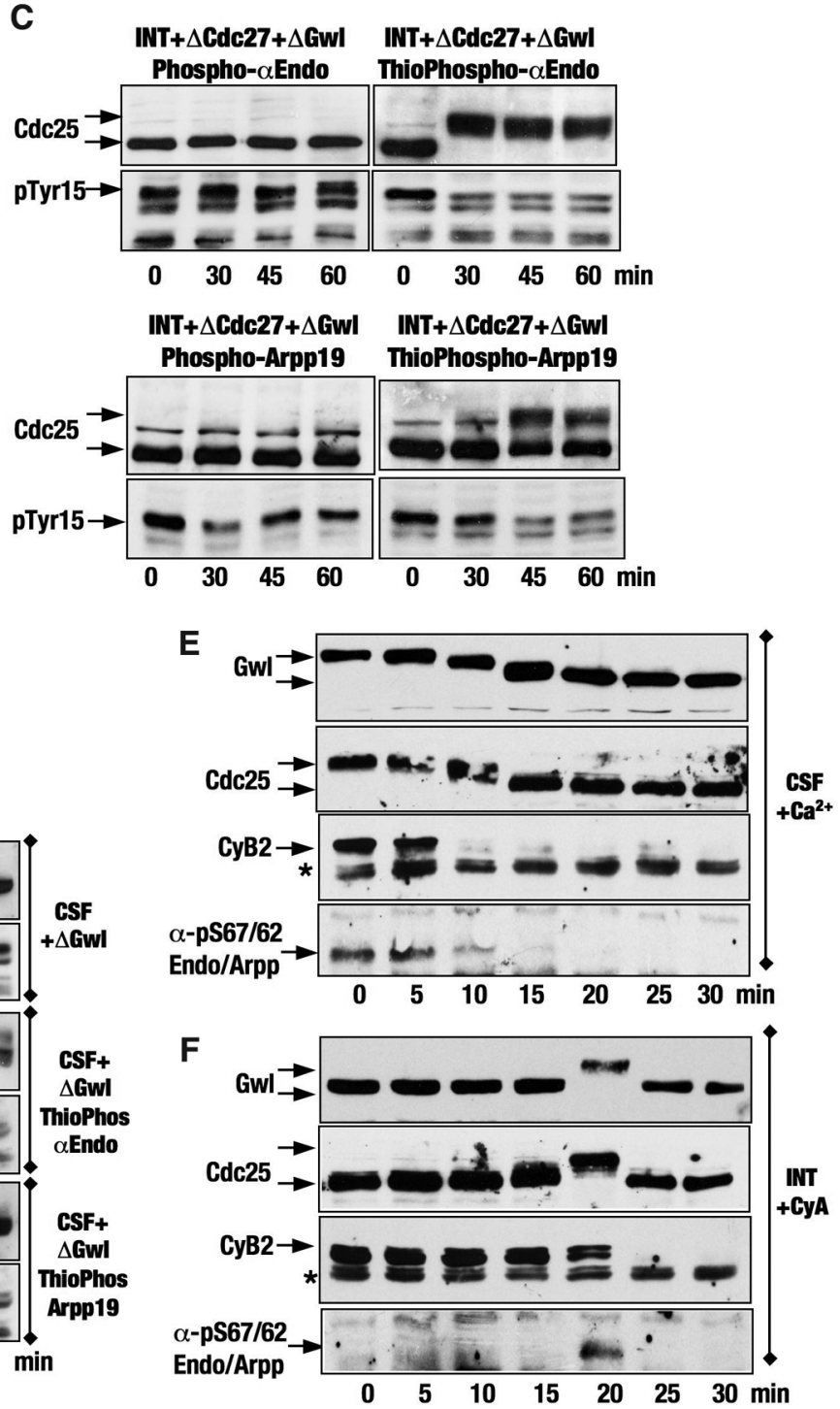

Fig. 2. Addition of thio-phosphorylated Arpp19 and $\alpha$-Endosulfine rescues the phenotype induced by Gwl depletion in interphase and mitotic egg extracts. (A) Interphase extracts were depleted of $\mathrm{Cdc} 27$ and supplemented at a final concentration of $170 \mathrm{ng} / \mu \mathrm{l}$ with the proteins GST-Arpp19 and GST- $\alpha$ Endosulfine phosphorylated or not in vitro by Gwl. The Gwl-phosphorylation mutants Arpp19-S62A and $\alpha$-Endosulfine-S67A were also used. (B) Mitotic entry was analyzed in interphase extracts that were depleted of Cdc27 and Cdc25 and supplemented with phospho-Arpp19 and phospho- $\alpha$-Endosulfine (final concentration of $170 \mathrm{ng} / \mu \mathrm{l}$ ). The phosphorylation of cyclin B-Cdc2 substrates was analyzed with an antibody recognizing the phospho-Serine
Cdk consensus motif. (C) Interphase extracts were depleted of Cdc27 and Gwl and supplemented with phosphorylated or thio-phosphorylated Arpp19 and $\alpha$-Endosulfine. (D) Mitotic extracts (CSF) were supplemented with thiophosphorylated Arpp19 or $\alpha$-Endosulfine and depleted of Gwl. (E) Mitotic exit was induced in CSF extracts by the addition of $\mathrm{Ca}^{2+}$, and the amounts of cyclin B2 and phosphorylation of Cdc25, Gwl, and the Gwl-specific sites of Arpp19 and $\alpha$-Endosulfine were analyzed. (F) Interphase extracts were supplemented with cyclin A (final concentration of $60 \mathrm{nM}$ ), and the kinetics of the phosphorylation of the indicated proteins as well as the levels of cyclin B2 were analyzed. 
A

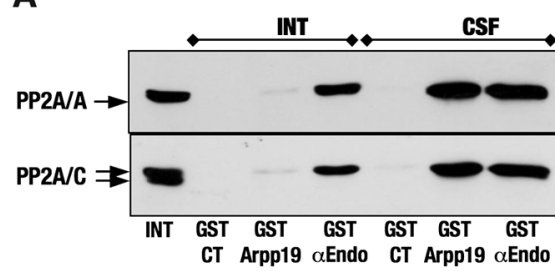

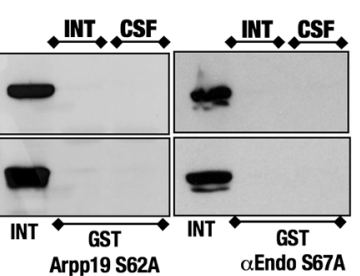

C

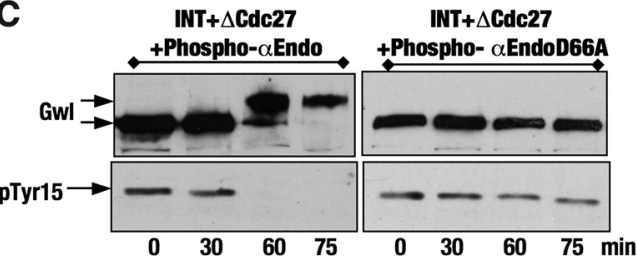

B

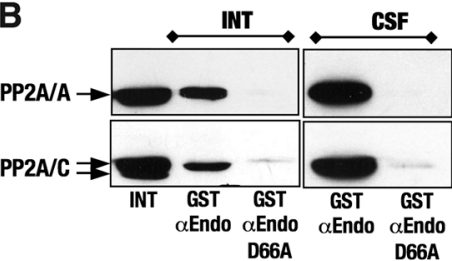

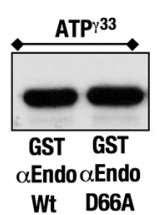

D

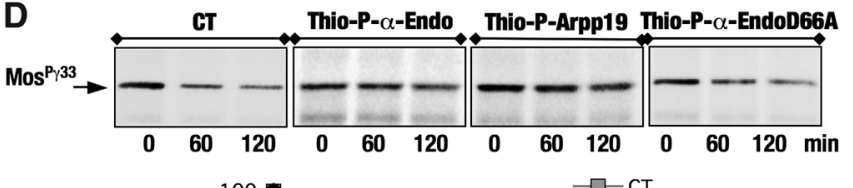

Fig. 3. Inhibition of PP2A by phosphorylated Arpp19 and $\alpha$-Endosulfine. (A) Interphase or mitotic extracts were supplemented with GST-Sepharose, GST-Arpp19-Sepharose, GST- $\alpha$ Endosulfine-Sepharose, the GST-Arpp19 (S62A)-Sepharose, or GST- $\alpha$-Endosulfine (S67A)Sepharose, and the binding of PP2A subunits A and C was analyzed by GST precipitation and Western blot. (B) Similar to (A) except for the addition of GST- $\alpha$-EndosulfineSepharose and GST- $\alpha$-Endosulfine (D66A)-Sepharose. Lower panels show the in vitro phosphorylation of the $\alpha$-Endosulfine (D66A) mutant by Gwl. (C) Effect of the phospho- $\alpha$ Endosulfine (D66A) mutant on mitotic entry in interphase extracts. (D) PP2A complex obtained by immunoprecipitation from interphase extracts was left untreated (CT) or was incubated for $10 \mathrm{~min}$ with $5.3 \mu \mathrm{g}$ of either thio-phospho-GST-Arpp19, thio-phospho-GST$\alpha$-Endosulfine, or thio-phospho-GST- $\alpha$-Endosulfine (D66A) and then mixed with phospho-

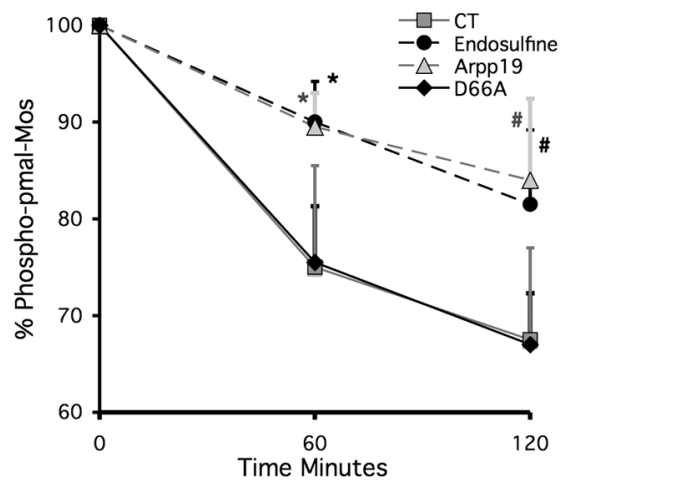
radiolabeled myelin basic protein-c-Mos fusion protein. At the indicated times, $15 \mu$ l of supernatant was analyzed by SDS-polyacrylamide gel electrophoresis and autoradiography. Gels were scanned by a Typhoon Scanner and quantified with Image software. Statistical analysis of the results obtained from two different independent experiments was performed with an unpaired Student's $t$ test. The percentage of phosphorylated c-Mos present at each time was expressed as the mean \pm SD. Statistical differences between control versus either Arpp19 or $\alpha$-Endosulfine at the two time points are indicated. ${ }^{*} P<0.03 ; \# P<0.04$

Fig. 4. Endogenous Arpp19 but not $\alpha$-Endosulfine is required to promote mitotic entry. (A) Interphase extracts were depleted of $\mathrm{Cdc} 27$ and either Arpp19 or $\alpha$ Endosulfine and then supplemented with cyclin A. (B) Mitotic extracts were depleted of Arpp19 or $\alpha$-Endosulfine, and the mitotic state was analyzed. (C) Mitotic extracts were depleted of $\mathrm{Cdc} 27$ and Arpp19 and 20 min later were $(+O A)$ or were not $(-O A)$ supplemented with okadaic acid (final concentration of $0.7 \mu \mathrm{M}$ ), and the mitotic state was analyzed. (D) HeLa cells were transfected or not (Lipo) with scrambled small interfering RNA (siRNA) (SC) or 50 or $100 \mathrm{nM}$ of Arpp19 siRNA for 24 hours, then synchronized by thymidine and released into nocodazole $(50 \mathrm{ng} / \mathrm{ml})$ for 10 hours. The percentage of mitotic cells was measured by a two-dimensional fluorescence-activated cell sorting (propidium iodide-antibody against phospho serine (dk) and expressed as the mean \pm SD. Statistical differences between scrambled and either 50 or $100 \mathrm{nM}$ Arpp19 siRNA are indicated ${ }^{*} P<8.03 \times 10^{-6}$ and ${ }^{*} P<$ $1.45 \times 10^{-6}$ ). The cellular levels of endogenous Arpp19 at each condition are shown.

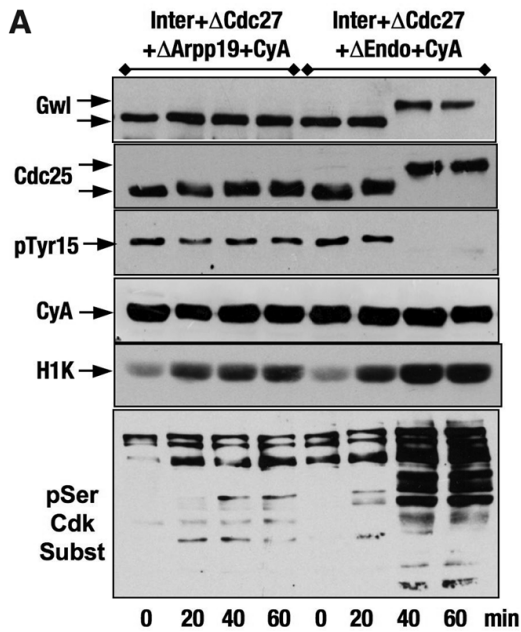

C

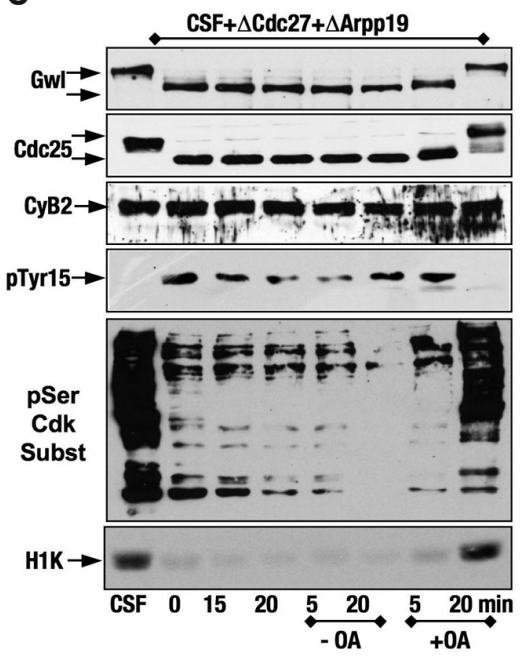

B

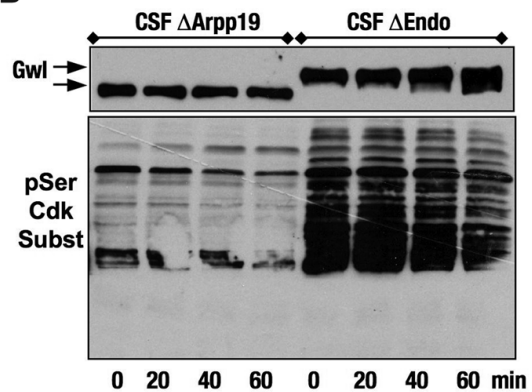

D

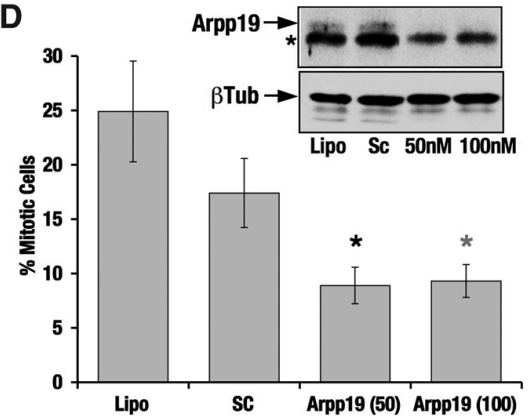


PP2A, although it was normally phosphorylated by Gwl at residue S67 (Fig. 3B). Moreover, the addition of this mutant previously phosphorylated by Gwl into interphase extracts did not induce mitotic entry (Fig. 3C), consistent with the idea that Gwl may inhibit PP2A by promoting the binding of its inhibitors, Arpp19 and $\alpha$-Endosulfine.

We tested directly the effect of Arpp19 and $\alpha$-Endosulfine on PP2A activity obtained from immunoprecipitated CSF extracts by assessing dephosphorylation of the cyclin B-Cdc2 substrate $\mathrm{c}-\mathrm{Mos}$ in vitro in the presence or in the absence of thio-phosphorylated-Arpp19, thio-phosphorylated$\alpha$-Endosulfine, and thio-phosphorylated- $\alpha$ Endosulfine D66A. Thio-phosphorylated-Arpp19 and thio-phosphorylated- $\alpha$-Endosulfine significantly decreased dephosphorylation of c-Mos by PP2A. Thio-phosphorylated- $\alpha$-Endosulfine mutant D66A, which does not bind PP2A, had no effect (Fig. 3D).

To test the physiological role of Arpp19 and $\alpha$-Endosulfine, we depleted interphase extracts of $\mathrm{Cdc} 27$ and then depleted them with specific antibodies against Arpp19 or $\alpha$-Endosulfine (fig. S2), and finally supplemented them with cyclin A. Depletion of Arpp19, but not of $\alpha$-Endosulfine (Fig. 1C), completely inhibited entry into mitosis (Fig. 4A) - a phenotype that was rescued by adding back this thio-phosphorylated protein (fig. S3). Similarly, only depletion of Arpp19 from CSF ex- tracts caused rapid exit of mitosis (Fig. 4B). This exit appeared to be mediated by a reactivation of PP2A because the inhibition of PP2A with okadaic acid caused these extracts to reenter mitosis (Fig. 4C). Thus, despite the inhibitory effects of both Arpp19 and $\alpha$-Endosulfine on PP2A, only Arpp19 appears to regulate mitotic entry and exit in Xenopus egg extracts. Consistent with this, only Arpp19 is phosphorylated during mitosis (fig. S2) and, despite the presence of larger amounts of endogenous $\alpha$-Endosulfine than endogenous Arpp19 in Xenopus egg extracts, only Arpp19 was identified in our biochemical analysis.

To investigate whether this mechanism was also conserved in human cells, we depleted Arpp19 from human cervical cancer (HeLa) cells using two different sequences of small interfering RNA (siRNA) (fig. S4). Depletion of Arpp19 reduced the number of mitotic cells by $50 \%$ compared to that in cells treated with a scramble siRNA, suggesting that Arpp19 also promotes mitotic entry in human cells (Fig. 4D and fig. S4).

Our results demonstrate an essential role of Arpp19 in regulating mitosis and provide a mechanism by which Gwl can influence cell cycle control through regulation of PP2A. Whether Arpp19 might be dephosphorylated at mitotic exit remains to be elucidated. Perhaps other physiological pathways might be regulated by $\alpha$-Endosulfinedependent inhibition of PP2A.

References and Notes

1. M. Jackman, C. Lindon, E. A. Nigg, J. Pines, Nat. Cell Biol. 5, 143 (2003).

2. P. V. Castilho, B. C. Williams, S. Mochida, Y. Zhao, M. L. Goldberg, Mol. Biol. Cell 20, 4777 (2009).

3. S. Mochida, S. Ikeo, J. Gannon, T. Hunt, EMBO J. 28, 2777 (2009)

4. S. Vigneron et al., EMBO J. 28, 2786 (2009).

5. J. Q. Wu et al., Nat. Cell Biol. 11, 644 (2009).

6. A. Burgess et al., Proc. Natl. Acad. Sci. U.S.A. 107 12564 (2010).

7. V. Archambault, X. Zhao, H. White-Cooper, A. T. Carpenter, D. M. Glover, PLoS Genet. 3, e200 (2007).

8. D. Bataille et al., Cell. Mol. Life Sci. 56, 78 (1999).

9. L. Gros et al., Diabetologia 45, 703 (2002).

10. J. R. Von Stetina et al., Development 135, 3697 (2008).

11. T. Lorca et al., J. Cell Sci. 123, 2281 (2010).

12. We thank L. Gros and A. Virsolvy for providing the pBKS-Arpp19, pBKS- $\alpha$-Endosulfine constructs, and antibodies to human $\alpha$-Endosulfine and T. Hunt and S. Mochida for the antibody to phospho567/62 Endo-Arp. This work was supported by the Ligue Regionale Contre le Cancer (Comité du Gard) and the "Association pour la Recherche sur le Cancer". A.B. and E.B. are "Fondation pour la Recherche Medicale" and

"Ligue Nationale Contre le Cancer" fellows, respectively. There is a patent pending that pertains to results presented in this paper.

\section{Supporting Online Material}

www.sciencemag.org/cgi/content/full/330/6011/1673/DC1 Materials and Methods

Figs. $\mathrm{S} 1$ to $\mathrm{S} 4$

References

27 August 2010; accepted 18 October 2010 $10.1126 /$ science. 1197048

structure involved in natural reward-related behaviors and responses to drugs such as cocaine $(15-19)]$ is required for reward learning, but novel studies of molecular ablation of cholinergic interneurons within the NAc instead have reported enhanced reward learning (20). Cholinergic interneurons within the NAc are particularly intriguing because they constitute less than $1 \%$ of the local neural population (21), yet they project throughout the NAc and provide its only known cholinergic input (22). Relevant cholinergic receptors are expressed locally, and nicotinic and muscarinic pharmacological agonists can exert complex influences on medium spiny neurons (MSNs, which represent $>95 \%$ of the local neuronal population and constitute the output of the NAc) (23-25). However, the net effect (if any) of the cholinergic interneurons on any aspect of NAc physiology or behavior is unknown.

We undertook an optogenetic approach to resolve this question by selectively driving or blocking action potential firing in these cells. To

A cetylcholine is an important and widely studied neurotransmitter, which acts on a variety of receptors and target cells (1-5). Pharmacological and genetic studies have elucidated the complex and often opposing influences of the individual subtypes of muscarinic and nicotinic acetylcholine receptors on numerous biological processes, but no study has yet resolved the question of the causal role of cholinergic neurons themselves within a central nervous system tissue (6-11). Addressing such a question would require a novel paradigm for selective and temporally precise control (activation and inhibition) of cholinergic neurons within living mammalian tissues, because previous investigations have resulted in contradictory findings linked to challenges with specificity and temporal resolution. For example, elegant in vivo pharmacological approaches have shown (12-14) that cholinergic transmission in the nucleus accumbens (NAc) [a
${ }^{1}$ Department of Bioengineering, Stanford University, Stanford, CA 94305, USA. ²Department of Neurosurgery, Stanford University, Stanford, CA 94305, USA. ${ }^{3}$ Department of Psychiatry and Behavioral Sciences, Stanford University, Stanford, CA 94305, USA. ${ }^{4}$ Howard Hughes Medical Institute, Stanford University, Stanford, CA 94305, USA. ${ }^{5}$ CNC program, Stanford University, Stanford, CA 94305, USA.

*These authors contributed equally to this work. †To whom correspondence should be addressed. E-mail: deissero@stanford.edu 